\title{
Consenso de Aspiração de Crianças com Tubo Endotraqueal da Sociedade Brasileira de Enfermeiros Pediatras (SOBEP) - 2018
}

\author{
Autores do Consenso: \\ Viviane Martins da Silva ${ }^{1}$, Gabrielle Silveira Alves Sampaio ${ }^{2}$, Nirla Gomes Guedes ${ }^{2}$, Denise Miyuki Kusahara ${ }^{3}$, \\ Marta José Avena ${ }^{3}$, Patrícia Kuerten Rocha ${ }^{4}$
}

Realização: Comissão Permanente de Assistência da Sociedade Brasileira de Enfermeiros Pediatras (SOBEP).

Coordenadoras: Viviane Martins da Silva (Coordenadora da Comissão Permanente de Assistência) e Myriam

Aparecida Mandetta (Presidente da SOBEP).

Nota: Estas diretrizes se prestam a informar e não a substituir o julgamento clínico da equipe multidisciplinar que, em última análise, deve determinar a intervenção apropriada para seus pacientes.

Instituição correspondente: Sociedade Brasileira de Enfermeiros Pediatras (SOBEP)/ Rua Napoleão de Barros, 275, 04024-001, Vila Clementino, São Paulo, SP Brasil. / E-mail: sobep@sobep.org.br.

DOI: http://dx.doi.org/10.31508/1676-3793201800017

\section{Como citar:}

Silva VN, Sampaio GS, Guedes NG, Kusahara DM, Avena MJ, Rocha PK. Consenso de aspiração de crianças com tubo endotraqueal da Sociedade Brasileira de Enfermeiros Pediatras (SOBEP) - 2018. Rev Soc Bras Enferm Ped. 2018;18(2):109-15.

${ }^{1}$ Departamento de Enfermagem, Universidade Federal do Ceará, Fortaleza, CE, Brasil

2 Universidade Federal do Ceará, Fortaleza, CE, Brasil

${ }^{3}$ Escola Paulista de Enfermagem, Universidade Federal de São Paulo, São Paulo, SP, Brasil

${ }^{4}$ Universidade Federal de Santa Catarina, Florianópolis, SC, Brasil

\section{Introdução}

A aspiração endotraqueal constitui uma técnica manual de inserção de um cateter estéril flexível através do tubo endotraqueal e aplicação de pressão negativa para remoção de secreções pulmonares e traqueobrônquicas. ${ }^{(1,2)}$

A aspiração endotraqueal é um procedimento comumente realizado. Esta tem por finalidades remover secreção e prevenir obstrução de vias aéreas. Dessa forma, diminui o risco de atelectasia ao permitir a otimização da oxigenação e da ventilação. ${ }^{(3,4)}$

A intubação endotraqueal e a ventilação mecânica são tratamentos necessários a neonatos, infantes e crianças com insuficiência respiratória relacionada a uma variedade de condições clínicas como pneumopatias, cardiopatias congênitas, choque, cirurgias com anestesia geral etc. ${ }^{(1)}$

A presença de via aérea artificial pode levar ao aumento das secreções respiratórias, à irritação tecidual e a inibição da ação mucociliar e do reflexo da tosse. ${ }^{(5)}$

Neste sentido, a aspiração de tubo endotraqueal constitui importante procedimento para controle das vias aéreas. Dada a complexidade clínica dos pacientes entubados e sob ventilação mecânica, a Sociedade Brasileira de Enfermeiros Pediatras (SOBEP) orienta que a aspiração de tubo endotraqueal seja um procedimento de competência do enfermeiro dentro da equipe de enfermagem. Esta 
técnica também é de execução compartilhada com outros membros da equipe multidisciplinar.

\section{Descrição do método de coleta de evidência utilizado}

A revisão bibliográfica que permitiu a identificação das evidências científicas e proporcionou a discussão dos membros especialistas da SOBEP foi realizada nas bases de dados SCOPUS, CINAHL, LILACS, PEDro, COCHRANE a partir da combinação de descritores e palavras-chave MeSH: Suction AND pediatrics; Suctioning endotracheal AND pediatrics; Suction AND normal saline AND pediatrics; Suction AND sodium chloride AND pediatrics; Suction cateter AND pediatrics; Suction AND clinical criteria AND pediatrics; Airway AND sputum AND suction AND pediatrics; Airway secretion AND suction AND pediatrics; Respiratory aspiration AND pediatrics. A seleção dos documentos e a extração dos dados foram realizadas por duplas de revisores, trabalhando de forma independente. A avaliação da qualidade dos artigos foi realizada com a utilização de guias internacionais que orientam os autores na preparação das revisões, especificamente AMSTAR e STROBE.

\section{Nível de evidência}

1 ++ Meta-análises de alta qualidade, revisões sistemáticas de Ensaios clínicos ou Ensaios clínicos com um risco muito baixo de viés.

1+ Meta-análises bem conduzidas, revisões sistemáticas de Ensaios clínicos ou Ensaios clínicos com baixo risco de viés.

1- Meta-análises, revisões sistemáticas ou Ensaios clínicos, ou Ensaios clínicos com alto risco de viés.

2 ++ Revisões sistemáticas de alta qualidade de estudos de caso-controle ou de coorte, ou de coorte de estudos com um risco muito baixo viés.

2+ Caso-controle bem conduzido ou estudos de coorte com baixo risco de viés, ou moderada probabilidade de que a relação seja causal.

2- Estudos de caso-controle ou coorte com alto risco de viés.

3 Estudos não analíticos, por exemplo, relatos de casos, série de casos.

4 Opinião de Especialista.

\section{Grau de recomendação}

A: Pelo menos uma meta-análise sistemática, revisão sistemática ou ensaio clínico classificado como 1++ e diretamente aplicável ao alvo à população alvo, ou uma revisão sistemática de ensaio clínico ou um conjunto de evidências principalmente de estudos classificados como 1+ diretamente aplicável à população e demonstrando consistência global dos resultados.

B: Um conjunto de evidências incluindo estudos classificados como 2++ diretamente aplicável à população-alvo e demonstrando consistência geral dos resultados ou evidência extrapolada de estudos classificados como $1++$ ou $1+$.

C: Um conjunto de evidências incluindo estudos avaliados como 2+ diretamente aplicável à população -alvo e demonstrando consistência geral dos resultados ou evidência extrapolada de estudos classificados como $2++$.

D: Nível de evidência 3 ou 4 ou evidência extrapolada de estudos classificados como $2+$.

\section{Objetivo deste consenso}

Orientar o enfermeiro pediatra na tomada de decisão sobre a aspiração endotraqueal e guiar a construção de protocolos de cuidado para uniformização da assistência e adaptação das recomendações à realidade local.

\section{População alvo}

Enfermeiros pediatras, gestores de enfermagem, acadêmicos de enfermagem e demais envolvidos direta ou indiretamente no cuidado à criança com indicação de aspiração endotraqueal.

\section{Reunião de consenso}

A reunião de consenso aconteceu em maio de 2018 na Escola Paulista de Enfermagem da UNIFESP. O resultado das revisões realizadas foi apresentado pela coordenadora a professores, gerentes e enfermeiros membros da SOBEP para discussão das evidências e definição das recomendações. 


\section{Recomendações:}

\section{Indicação}

A aspiração endotraqueal é um procedimento com potenciais efeitos adversos como sangramento, atelectasia, hipoxemia, instabilidade cardiovascular, elevação da pressão intracraniana, lesão da mucosa traqueal. ${ }^{(6)}$ Esses efeitos têm sido relacionados à aspiração de ar pelas vias aéreas e à estimulação vagal devido à introdução do cateter e à pressão negativa gerada na via aérea. $^{(7)} \mathrm{O}$ procedimento ainda causa dor. Esta, quando instalada, potencializa outros eventos adversos por aumentar as demandas do sistema cardiorrespiratório e elevar os níveis de pressão intracraniana. ${ }^{(8,9)}$ Devido ao risco e potenciais efeitos adversos, não se recomenda que a aspiração endotraqueal seja realizada rotineiramente, devendo ser indicada somente quando há sinais de secreção. ${ }^{(6)}$

A realização da aspiração somente quando necessária requer que enfermeiros avaliem a presença de indicadores clínicos sugestivos de obstrução de vias aéreas. A literatura aponta indicadores clínicos e comportamentais associados à fisiopatologia e à estabilidade clínica que podem indicar a presença de vias aéreas obstruídas. ${ }^{(10)}$

Entre os indicadores clínicos, foram considerados para aspiração em recém-nascidos entubados: secreções visíveis no tubo endotraqueal, secreções audíveis, sons respiratórios diminuídos, excursão respiratória diminuída, alteração nos gases sanguíneos, alterações na frequência respiratória, bradicardia, taquicardia, agitação sem outras causas e aumento do pico de pressão respiratória. A necessidade de aspiração deve ser preferencialmente avaliada por meio da ausculta. ${ }^{(2)}$

Além de permitir a indicação da aspiração, o monitoramento de indicadores clínicos antes, durante e depois de sua realização tornará o procedimento mais seguro. Entre os indicadores a serem monitorados, a literatura inclui: sons respiratórios; $\mathrm{SpO}$; coloração da pele; frequência respiratória; padrão respiratório; variáveis hemodinâmicas como frequência cardíaca, pressão arterial, ritmo cardíaco e PIC; características da secreção aspirada como cor, volume, consistência e odor; características da tosse; parâmetros ventilatórios, como pico de pressão inspiratória e pressão de platô; fluxo; volume exalado; e FiO2.(11,12)
Com base na literatura disponível, a SOBEP recomenda que os enfermeiros pediatras não realizem a aspiração endotraqueal de rotina. Recomenda ainda que os profissionais apliquem métodos propedêuticos como a inspeção e palpação para avaliar indicadores clínicos que caracterizam sofrimento respiratório por obstrução de vias aéreas. Além de alterações dos sons pulmonares, indicadores clínicos como retrações intercostais, batimento de aletas nasais, agitação, bem como dados de oximetria de pulso como saturação < $90 \%$ podem orientar a tomada de decisão do enfermeiro pediatra para indicar e monitorar respostas ao procedimento.

\section{Contraindicação}

Não há contraindicações absolutas para a aspiração endotraqueal devido à natureza obrigatória do procedimento. ${ }^{(13)} \mathrm{Na}$ prática clínica, a aspiração endotraqueal é evitada em recém-nascidos 15 a 30 minutos após a administração do surfactante. ${ }^{(14)}$ Em casos especiais, como a hipertensão intracraniana descontrolada, medidas adicionais para controle da pressão intracraniana (PIC) devem ser adotadas antes da aspiração endotraqueal em recém-nascidos. ${ }^{(2)}$

A SOBEP endossa as recomendações da literatura para indicação e contraindicação da aspiração endotraqueal. Ressalta ainda outras condições especiais que necessitarão de avaliação criteriosa do enfermeiro pediatra para realização de medidas adicionais e complementares à aspiração como a prematuridade extrema e a fibrose cística.

\section{Normas de biossegurança}

Para o desempenho da aspiração endotraqueal, a literatura recomenda a proteção de olhos, nariz e boca do profissional com o uso de máscara facial e óculos de proteção. Os profissionais devem ainda vestir avental e luvas estéreis, além de realizar a higiene das mãos antes e depois do procedimento. ${ }^{(2)}$ Para aumentar a segurança do procedimento, orienta-se que a aspiração endotraqueal seja realizada por pelo menos duas pessoas..$^{(2,15)}$

A SOBEP endossa a necessidade do uso de equipamentos de proteção individual para manter a segurança do procedimento. 


\section{Características da sonda de aspiração}

O cateter deve ser estéril, flexível, com vários orifícios laterais e um frontal. ${ }^{(16)} \mathrm{O}$ diâmetro do cateter deve ser menor que $50 \%$ do diâmetro interno do tubo endotraqueal. ${ }^{(15,16)}$ Entretanto, diante de tubos endotraqueais com diâmetro interno de 2,5 ou 3,0 mm, um cateter de sucção de tamanho recomendado ocluirá $75 \%$ do lúmen do tubo endotraqueal. ${ }^{(4,16)}$ Um cateter de aspiração com um diâmetro externo que oclua menos de $40 \%$ do diâmetro interno do tubo pode ser insuficiente para limpar as secreções, levando ao aumento do número de repetições. ${ }^{(6)}$ Por outro lado, cateter com diâmetro que oclua mais que $70 \%$ da luz do tubo são difíceis de serem introduzidos. ${ }^{(16)}$

A seleção do tamanho do cateter deve ser feita considerando tanto o tamanho do tubo quanto a consistência da secreção. Recomenda-se o uso do menor cateter de aspiração capaz de remover adequadamente as secreções, visto que o tamanho do cateter provavelmente tem uma influência maior na perda de volume pulmonar em relação à pressão negativa de aspiração. ${ }^{(12,15)}$

A SOBEP endossa a recomendação de que a seleção do cateter de aspiração deve ser o menor possível, avaliadas informações como tamanho do tubo endotraqueal e características da secreção. Ressalta ainda que, além de estéril e flexível, o cateter deve ser transparente e incolor para permitir a visualização das caraterísticas da secreção aspirada.

\section{Tempo de aspiração}

A duração do procedimento pode aumentar a pressão negativa e reduzir os volumes pulmonares. Assim, a duração da aspiração está associada a severidade dos efeitos adversos. Autores também recomendam uma duração de aspiração entre 10 e 15 segundos para adultos e cinco segundos ou menos para pacientes pediátricos e neonatos. ${ }^{(16)}$

Independentemente da idade do paciente, o tempo total do procedimento deve limitar-se a no máximo 15 segundos. O paciente deve estar monitorado por oximetria de pulso durante e no seguimento do procedimento. ${ }^{(2)}$

A SOBEP endossa a recomendação para um tempo total de aspiração de no máximo 15 segundos.

\section{Pressão negativa de aspiração}

A pressão negativa exercida na ponta e ao longo do tubo endotraqueal é influenciada por variáveis como o tamanho do cateter de aspiração e do tubo endotraqueal, da duração da sucção, bem como do volume e da viscosidade das secreções. ${ }^{(15)}$

A literatura destaca que uma pressão negativa é gerada dentro dos pulmões somente enquanto o ar sai do cateter de aspiração. ${ }^{(15,16)}$ Deste modo, assim que as secreções são aspiradas para dentro do cateter, a pressão intrapulmonar retorna ao nível atmosférico e a perda de volume pulmonar é interrompida. Neste sentido, é necessária criteriosa avaliação da criança para indicação do procedimento, visto que a realização da aspiração endotraqueal na ausência de secreções potencializa os efeitos da pressão negativa, podendo causar atelectasias significativas. ${ }^{(16)}$

A literatura aponta que, em neonatos, pressões até $200 \mathrm{mmHg}$ foram aplicadas para aspirar secreções grossas. ${ }^{(16)}$ A literatura recomenda, no entanto, que a pressão negativa de aspiração deve permanecer entre 80 e 100mmHg.(2) Recomenda-se ainda que a pressão de aspiração seja verificada pela oclusão do final da sonda antes de iniciar o procedimento. ${ }^{(7)}$

A SOBEP endossa a recomendação a pressão negativa de aspiração deve permanecer entre 80 e $100 \mathrm{mmHg}$. Recomenda ainda que a seleção da pressão negativa de aspiração deve ser fundamentada em informações como tamanho do cateter de aspiração e do tubo endotraqueal, além das características da secreção.

\section{Número de repetições}

Além de preocuparem-se com a frequência de realização da aspiração endotraqueal, alguns estudos discutem o número de inserções do cateter necessário para remoção efetiva das secreções com um mínimo de riscos para a criança. ${ }^{(2,16)}$

O tamanho do cateter e a pressão negativa influenciam o número de repetições do procedimento. A quantidade de inserções do cateter durante a aspiração endotraqueal tem sido relacionada a probabilidade de complicações, como trauma mucoso, hipoxemia, laringoespasmo, broncoespasmo, traqueíte necrosante, infecção e desconforto, além de aumentar a probabilidade de barotrauma. ${ }^{(11,12)}$ 
Dadas as potenciais complicações, a literatura recomenda que o número de repetições não deva exceder três inserções. Os autores ainda orientam que o profissional deve esperar o retorno dos indicadores monitorados ao nível anterior ao procedimento. (2,11,15) $^{-1}$

A SOBEP endossa a recomendação de no máximo três inserções do cateter durante o procedimento de aspiração do tubo endotraqueal. Recomenda ainda avaliação clínica criteriosa entre as inserções para a tomada de decisão de retorno e continuidade do procedimento.

\section{Instilação de solução salina}

A solução salina tem sido usada no procedimento de aspiração endotraqueal para estimular a tosse e favorecer a mobilização de secreção. Esta prática pode ser ainda utilizada para lubrificação das paredes do tubo endotraqueal, favorecendo a entrada e a progressão do cateter endotraqueal. ${ }^{(6)}$ Alguns profissionais instilam solução salina para fluidificar as secreções. Entretanto, estudos têm indicado que muco e água são imiscíveis in vitro e mantém suas fases separadas, mesmo após agitação vigorosa. ${ }^{(17,18)}$

Em revisão das evidências científicas, autores encontraram que, do volume de solução salina instilada, entre $10,7 \%$ e $18,7 \%$ foi recuperada pelo cateter de aspiração. Entretanto, o restante permaneceu no tubo traqueal e no sistema respiratório, diminuindo o diâmetro do tubo, elevando a resistência na via aérea ao fluxo aéreo e reduzindo o oxigênio disponível nos pulmões para a troca gasosa. ${ }^{(19)}$

Em um estudo envolvendo 24 crianças criticamente doentes, autores verificaram que os pacientes que, tiveram solução salina instilada, apresentaram dessaturação de oxigênio significativamente maior 1 e 2 min após a aspiração em comparação com os pacientes que não a receberam. ${ }^{(20)}$

A literatura apresenta que a instilação aumenta a probabilidade de arritmia cardíaca, hipoxemia, atelectasia, broncoespasmo, infecção. A instilação também esteve associada a redução da saturação de oxigênio nos primeiros minutos após o procedimento. ${ }^{(3,20)}$

Em crianças, a instilação foi benéfica para manutenção de perviabilidade do tubo com diâmetro de 2,5 $\mathrm{mm}$. Instilações entre 0,25 e $0,5 \mathrm{ml}$ não estiveram associadas a alterações de indicadores entre grupos de neonatos. Já instilações entre 0,5 e 2,0 ml estiveram relacionados a queda da saturação. Para secreções tenazes, $0,1-0,2 \mathrm{ml}$ de solução salina a $0,9 \% / \mathrm{kg}$ de peso corporal pode ser instilado antes da aspiração. ${ }^{(16)}$

A literatura recomenda evitar a rotina de instilação de solução salina não, entretanto aconselha-se uma umidificação adequada das vias aéreas. ${ }^{(3,15,16)}$

A SOBEP não recomenda a instilação de solução salina antes ou durante o procedimento de aspiração.

\section{Hiperoxigenação}

A hiperoxigenação constitui o aumento do oxigênio inspirado imediatamente antes do início da aspiração endotraqueal com a finalidade reduzir o nível de hipoxemia. ${ }^{(6)} \mathrm{A}$ administração de oxigênio em excesso em curto período de tempo tem sido associada à hipercapnia, atelectasia por absorção, reposta inflamatória, perda de volume pulmonar.

Em prematuros, a administração de oxigênio a $100 \%$ é evitada por causa dos possíveis efeitos deletérios da hiperóxia (Ex.: hipercapnia, atelectasia por absorção, retinopatia da prematuridade, alterações alveolares e traqueobrônquicas, parênquima pulmonar e, principalmente, estresse oxidativo). Nestes pacientes, um aumento sustentado de $10 \%$ na $\mathrm{FIO}_{2}$ melhora o nível de oxigênio cerebral e saturação de oxigênio. ${ }^{(6,16)}$

Neonatos com redução clínica importante da $\mathrm{SpO}_{2}$ podem se beneficiar da hiperoxigenação antes do procedimento. ${ }^{(12)}$ Lactentes também parecem se beneficiar hiperoxigenação, visto haver possibilidade do desenvolvimento de bradicardia e apneia quando a hiperoxigenação antes do procedimento não é fornecida. ${ }^{(16)}$

Estudos sugerem que a taxa de $\mathrm{FiO} 2$ deve permanecer entre 10 e $20 \%$ acima do nível anterior à aspiração endotraqueal. ${ }^{(21,22)}$ Por outro, um aumento de $20 \%$ na $\mathrm{FiO} 2$ em relação àquela antes da aspiração é provavelmente tão eficaz quanto a hiperoxigenação com $100 \%$ de $\mathrm{FiO} 2$. . $^{(21-23)}$

A literatura recomenda que a elevação do valor basal deve ocorrer 30 a 60 segundos antes do procedimento até 1 minuto após. ${ }^{(2,12)}$

A SOBEP não recomenda a hiperoxigenação de rotina, dados os efeitos os efeitos adversos associados. Orienta ainda que casos individuais devem ser discutidos com a equipe multidisciplinar para tomada de decisão da aplicação de hiperoxigenação. 


\section{Profundidade da aspiração}

A aspiração endotraqueal profunda consiste na inserção do cateter de aspiração até que seja encontrada resistência pelo toque da carina. Considera-se aspiração endotraqueal superficial a inserção do cateter de aspiração até uma profundidade predeterminada. A determinação da profundidade de inserção do cateter será definida pelo somatório do comprimento da porção do tubo inserido e do adaptador. Por ser um procedimento minimamente invasivo, a tosse não é estimula e a manobra limpará apenas as secreções de dentro do lúmen. ${ }^{(6,24)}$

Uma aspiração endotraqueal profunda pode permitir o aumento de volume de muco aspirado durante o procedimento, além de manter a patência do tubo endotraqueal por maior período de tempo. ${ }^{(6)}$ Entretanto, já está bem documentado que aspirações endotraqueais profundas estão associadas a lesão de mucosa, estimulação e inflamação de tecido, significativa redução da saturação de oxigênio, pneumotórax. ${ }^{16,24-28)}$

Em estudos realizados com neonatos de alto risco, não houve diferenças significativas nas respostas da frequência cardíaca e saturação de oxigênio entre as aspirações profunda e superficial. ${ }^{(16)}$

A aspiração profunda não mostrou benefício superior à aspiração superficial. ${ }^{(16)}$

Dados os resultados inconclusivos e os potenciais efeitos adversos associados à profundidade, a SOBEP recomenda que enfermeiros pediatras evitem o uso da aspiração profunda.

\section{Sistema aberto / Fechado de aspiração}

Há dois métodos de aspiração de tubo endotraqueal: sistema aberto e sistema fechado. No sistema aberto, a criança é desconectada do ventilador para inserção do cateter de aspiração. Este sistema esteve associado a maior remoção de volume de secreções. ${ }^{(16)}$

No sistema fechado, o cateter encontra-se posicionado entre o tubo endotraqueal e o ventilador e pode ser usado várias vezes sem que haja a desconexão da criança ao ventilador. ${ }^{(6)}$ Embora a literatura traga resultados controversos quanto à superioridade do sistema fechado, este método esteve associado a menor tempo de procedimento e número de enfermeiros envolvidos, menores alterações de indicadores hemodinâmicas. ${ }^{(6,16)} \mathrm{A}$ aspiração pelo sistema fechado mostrou ser fisiologicamente mais bem tolerada em crianças e esteve relacionada a redução da dessaturação e bradicardia, a menor colonização de bactérias e a diminuição de sepse. ${ }^{(29-31)}$

A literatura encoraja a adoção de técnica estéril em aspirações em sistema aberto. ${ }^{(16)}$

A SOBEP acredita que ambos os métodos de aspiração apresentam vantagens e desvantagens. A SOBEP recomenda que a indicação do método deva fundamentar-se em avaliação clínica criteriosa e disponibilidade dos recursos necessários para ambos os métodos.

\section{Síntese das Recomendações da SOBEP para aspiração de crianças com tubo endotraqueal -2018}

\begin{tabular}{|c|c|}
\hline RECOMENDAÇÃO & $\begin{array}{c}\text { GRAU DE } \\
\text { RECOMENDAÇÃO }\end{array}$ \\
\hline $\begin{array}{l}\text { 1. Indicação: } \\
\text { A aspiração endotraqueal deve ser realizada somente } \\
\text { quando houver indicadores clínicos que sustentem sua } \\
\text { indicação. }\end{array}$ & $\mathrm{D}$ \\
\hline $\begin{array}{l}\text { 2. Contraindicação: } \\
\text { Não há contraindicações absolutas para a aspiração } \\
\text { endotraqueal. }\end{array}$ & $\mathrm{D}$ \\
\hline $\begin{array}{l}\text { 3. Norma de biossegurança: } \\
\text { Proteção de olhos, nariz e boca do profissional com o } \\
\text { uso de máscara facial e óculos de proteção. Uso de } \\
\text { avental e luvas estéreis. Realização da higiene das mãos } \\
\text { antes e depois do procedimento. }\end{array}$ & $A$ \\
\hline $\begin{array}{l}\text { 4. Características da sonda: } \\
0 \text { diâmetro do cateter deve ser menor que } 50 \% \text { do } \\
\text { diâmetro interno do tubo endotraqueal. }\end{array}$ & C \\
\hline $\begin{array}{l}\text { 5. Tempo de aspiração: } \\
0 \text { tempo total do procedimento deve limitar-se a no } \\
\text { máximo } 15 \text { segundos. }\end{array}$ & $\mathrm{C}$ \\
\hline $\begin{array}{l}\text { 6. Pressão negativa da aspiração: } \\
\text { A pressão negativa de aspiração deve permanecer entre } \\
80 \text { e } 100 \mathrm{mmHg} \text {. }\end{array}$ & D \\
\hline $\begin{array}{l}\text { 7. Número de repetições: } \\
\text { No máximo três inserções do cateter durante o procedimento }\end{array}$ & D \\
\hline $\begin{array}{l}\text { 8. Instilação de solução salina: } \\
\text { Não é recomendada a instilação de solução salina antes } \\
\text { ou durante o procedimento de aspiração. }\end{array}$ & $\mathrm{D}$ \\
\hline $\begin{array}{l}\text { 9. Hiperoxigenação: } \\
\text { Não é recomendada a hiperoxigenação de rotina. }\end{array}$ & $\mathrm{D}$ \\
\hline $\begin{array}{l}\text { 10. Profundidade da aspiração: } \\
\text { Recomenda-se que enfermeiros pediatras evitem o uso } \\
\text { da aspiração profunda. }\end{array}$ & D \\
\hline $\begin{array}{l}\text { 11. Sistema aberto/fechado: } \\
\text { Recomenda-se que a indicação do método deva } \\
\text { fundamentar-se em avaliação clínica criteriosa e } \\
\text { disponibilidade dos recursos necessários para ambos } \\
\text { os métodos. }\end{array}$ & $D$ \\
\hline
\end{tabular}




\section{Agradecimentos}

Aos autores e membros da SOBEP que participaram da reunião de Consenso para definição das recomendações contidas nesta publicação.

\section{Referências}

1. Bruschettini M, Zappettini S, Moja L, Calevo MG. Frequency of endotracheal suctioning for the prevention of respiratory morbidity in ventiled newborns. Cochrane Database Syst Rev. 2016;7(3):CD011493.

2. Gonçalves RL, Tsuzuki LM, Carvalho MG. Endotracheal suctioning in intubated newborns: an integrative literature review. Rev Bras Ter Intensiva. 2015;27(3):284-92.

3. Wang CH, Tsai JC, Chen SF, Su CL, Chen L, Lin CC, et al. Normal saline instillation before suctioning: A meta-analysis of randomized controlled trials. Aust Crit Care. 2017;30(5):260-5.

4. Morrow B, Futter M, Argent A. Effect of endotracheal suction on lung dynamics in mechanically-ventilated paediatric patients. Aust J Physiother. 2006;52(2):121-6.

5. Shah $\mathrm{C}$, Kollef $\mathrm{MH}$. Endotracheal tube intraluminal volume loss among mechanically ventilated patients. Crit Care Med. 2004;32(1):120-5.

6. Clifton-Koeppel R. Endotracheal Tube Suctioning in the Newborn:A Review of the Literature. Newborn Infant Nurs Rev. 2006;6(2):94-9.

7. Kiraly NJ, Tingay DG, Mills JF, Morley CJ, Copnell B. Negative tracheal pressure during neonatal endotracheal suction. Pediatr Res. 2008;64(1):29-33.

8. Cignacco E, Hamers JP, Stoffel L, van Lingen RA, Schütz N, Müller R, et al. Routine procedures in NICUs: factors influencing pain assessment andranking by pain intensity. Swiss Med Wkly. 2008;138(33-34):484-91.

9. Hadian ZS, Sabet RS. The Effect of Endotracheal Tube Suctioning Education of Nurses on Decreasing Pain in Premature Neonates. Iran J Pediatr. 2013;23(3):340-4.

10. Ireton J. Tracheostomy suction: a protocol for practice. Paediatr Nurs. 2007;19(10):14-18.

11. Gardner DL, Shirland L. Evidence-based guideline for suctioning the intubated neonate and infant. Neonatal Netw. 2009;28(5):281-302.

12. American Association for Respiratory Care. AARC Clinical Practice Guidelines Endotracheal suctioning of mechanically ventilated patients with artificial airways 2010. Respir Care. 2010;55(6):758-64.

13. Rosa FK, Roese CA, Savi A, Dias AS, Monteiro MB. Comportamento da mecânica pulmonar após a aplicação de protocolo de fisioterapia respiratória e aspiração traqueal em pacientes com ventilação mecânica invasiva. Rev Bras Ter Intensiva. 2007;19(2):170-5.
14. Rebello CM, Procianoy R, Freddi NA, Araújo KJ, Queirós Filho H, Mascaretti RS. I Consenso Brasileiro de Ventilação Mecânica em Pediatria e Neonatologia: uso do surfactante no recém-nascido. São Paulo: Associação de Medicina Intensiva Brasileira; 2012.

15. Pedersen CM, Rosendahl-Nielsen M, Hjermind J, Egerod I. Endotracheal suctioning of the adult intubated patient--what is the evidence? Intensive Crit Care Nurs. 2009;25(1):21-30.

16. Morrow BM, Argent AC. A comprehensive review of pediatric endotracheal suctioning: effects, indications, and clinical practice. Pediatr Crit Care Med. 2008;9(5):465-77.

17. Connolly MA. Mucolytics and the critically ill patient: help or hindrance? AACN Clin Issues. 1995;6(2):307-15.

18. Day T, Farnell S, Wilson-Barnett J. Suctioning: a review of current research recommendations. Intensive Crit. Care Nurs. 2002;18(2):79-89.

19. Halm MA, Krisko-Hagel K. Instilling normal saline with suctioning: beneficial technique or potentially harmful sacred cow? Am J Crit Care. 2008;17(5):469-72.

20. Ridling DA, Martin LD, Bratton SL. Endotracheal suctioning with or without instillation of isotonic sodium chloride solution in critically ill children. Am J Crit Care. 2003;12(3):212-9.

21. Walsh BK, Hood K, Merritt G. Pediatric airway maintenance and clearance in the acute care setting: how to stay out of trouble. Respir Care. 2011;56(9):1424-40.

22. Lookinland S,Appel PL. Hemodynamic and oxygen transport changes following endotracheal suctioning in trauma patients. Nurs Res. 1991;40(3):133-9.

23. Czarnik RE, Stone KS, Everhart CC Jr, Preusser BA. Differential effects of continuous versus intermittent suction on tracheal tissue. Heart Lung. 1991;20(2):144-51.

24. Bailey C1, Kattwinkel J, Teja K, Buckley T. Shallow versus deep endotracheal suctioning in young rabbits: pathologic effects on the tracheobronchial wall. Pedatrics. 1988;82(5):74651.

25. Brodsky L, Reidy M, Stanievich JF. The effects of suctioning techniques on the distal tracheal mucosa in intubated low birth weight infants. Int J. Otorhinolaryngol. 1987;14(1):1-14.

26. Ahn Y, Hwang T. The effects of shallow versus deep endotracheal suctioning on the cytological components of respiratory aspirates in high-risk infants. Respiration. 2003;70(2):172-8.

27. Yongmee A, Yonghoon J. The effects of the shallow and the deep endotracheal suctioning on oxygen saturation and heart rate in high-risk infants. Int J Nurs Stud. 2003;40(2):97104.

28. Gillies D, Spence K. Deep versus shallow suction of endotracheal tubes in ventilated neonates and young infants. Cochrane Database Syst Rev. 2011;6(7):CD003309.

29. Cordero L, Sananes M, Ayers LW. Comparison of a closed (Trach Care MAC) with an open endotracheal suction system in small premature infants. J. Perinatol. 2000;20(3):151-6.

30. Gunderson LP, Stoeckle ML. Endotracheal suctioning of the newborn piglet. West J Nurs Res. 1995;17(1):20-31.

31. Bloom BT, Craddock A, Delmore PM, Kurlinski JP, Voelker M, Landfish N, et al. Reducing acquired infections in the NICU: observing and implementing meaningful differences in process between high and low acquired infection rate centers. J. Perinatol. 2003;23(6):48992. 